\title{
Tracheobronchoscopic, cytological and microbiological results of tracheal and bronchial collapse in dogs")
}

\author{
HAKAN SALCI, MELIKE CETIN, SERPIL KAHYA*, AHMET AKKOC**, OZGE YILMAZ*, \\ UYGUR CANATAN, HILAL CESME, VILDAN ASLAN, CANAN ALTINCI, \\ MOHAMMED KHIDER*, ZEKI YILMAZ***, AHMET SAMI BAYRAM ${ }^{* * * *}$ \\ Department of Surgery, *Department of Microbiology, **Department of Pathology, \\ ***Department of Internal Medicine, Faculty of Veterinary Medicine, \\ ****Department of Thoracic Surgery, Faculty of Medicine, Uludağ University, 16059-Bursa, Turkey
}

Salci H., Cetin M., Kahya Demirbilek S., Akkoc A., Yilmaz O., Canatan U., Cesme H., Aslan V., Altinci C., Khider M., Yilmaz Z., Bayram A. S. Tracheobronchoscopic, cytological and microbiological results of tracheal and bronchial collapse in dogs

\section{Summary}

The aim of this study was to evaluate the tracheobronchoscopic, cytological and microbiological results of tracheal and bronchial collapse in dogs. In total, 8 dogs were included in the study. Clinically, tracheal palpations of the dogs were reflective of tracheal disease, and all dogs coughed on tracheal palpation. Vital parameters and hematological values of the dogs were within the normal ranges. Radiological views of the respiratory tracts and thorax were largely normal, but distinctive tracheal contours were noted in cases 3 and 6. Tracheobronchoscopy was performed under general anesthesia, and endoscopic findings (mucosal surfaces and color, prominent appearance of vessels, chondral ring abnormalities of the trachea, and the presence of bronchial and tracheal collapse) were scored. Bronchoalveolar lavage (BAL) was performed to collect samples for cytological and microbiological analysis. Five cases had tracheal collapse, and two cases had right bronchial collapse. Concurrent tracheal and right bronchial collapse were diagnosed in one case. Cytological results were not indicative of inflammation or infection, but Escherichia coli was isolated from case 2 (bronchial collapse) and case 3 (tracheal collapse). Antibiotic susceptibility results revealed that the organisms were susceptible to sulfamethoxazole/trimethoprim. Statistically, there were no significant differences between the cases in terms of total endoscopic scores. In conclusion, tracheal and/or bronchial collapse should only be diagnosed by tracheobronchoscopic examination. Cytological and microbiological analyses of the BAL fluid in these cases do not always provide valuable data for clinical practitioners.

Keywords: tracheobronchoscopy, cytology, microbiology, tracheal collapse, bronchial collapse, dog

In dogs, the most commonly encountered tracheal pathologies are tracheal stenosis, stricture, collapse, and tracheomalacia (softening of the tracheal cartilage) $(4,8,13,16)$. Tracheal collapse is a common cause of coughing in dogs, and it is seen in the cervical and/ or intrathoracic portion of the trachea $(4,7,8,10$, 15). Tracheal collapse and tracheomalacia are usually associated with congenital disease $(8,16)$, extrinsic compression or chronic inflammation, and they lead to airway weakness and inflammatory airway disease $(4,15,16)$. Bronchial collapse occurs in association with tracheal collapse $(1,8)$. It has been hypothesized

1) This study was performed with support from a scientific research project from Uludag University (Project no: BUAP(V)-2014/1). that bronchomalacia is a frequent finding of dogs with tracheal collapse and that it is associated with concurrent inflammatory airway disease, which may cause 2 bronchial collapse $(2,8)$. Little is known about the clinical and clinicopathological features of this disease (1).

Tracheal and bronchial collapses are easily identified by endoscopic examinations and are diagnosed by specific views $(7,8,10,14)$. These pathologies are graded by tracheobronchoscopic examination $(1,2,7$, $8,11,14)$, and intraluminal appearances are scored to demonstrate the severity of the disease $(2,4,11,14$, 15). Cytological evaluation of the bronchoalveolar lavage (BAL) fluid or tracheal brush cytology is made 
to analyze cellular characteristics of the airways and to provide evidence of inflammation or the presence of bacteria $(1,7,12)$.

The results of bacterial culture of tracheobronchial samples from dogs with tracheal collapse commonly contain Gram-negative, coliform and mixed populations $(5,7,12)$. Sample collection methods and cytological findings for the airways in tracheal collapse cases have not been described in the current literature $(7,8)$. Dogs with tracheal collapse often receive antimicrobials, but the role of bacterial infection of the airways in this disease remains unclear $(1,7,8)$. In addition, bacterial infection of the airways is generally accompanied by cytological evidence of suppurative inflammation (7). Therefore, cytological and microbiological results must be evaluated concurrently to characterize the pathological process of tracheal collapse $(7,14)$. This study reports tracheobronchoscopic findings in the airways, as well as cytological and microbiological results for BAL samples, in dogs with tracheal and/or bronchial collapse.

\section{Material and methods}

This study was conducted with permission from the Uludag University Local Ethic Committee (Decision no: 2013-01/05).

In 2016, 8 dogs of various breeds, ages and sexes were diagnosed with tracheal pathology in a project study. All dogs were presented at different times to Uludag University, Faculty of Veterinary Medicine, Department of Surgery Clinics and they were subjected to tracheobronchoscopy under general anesthesia following permission from the dogs' owners. In the history, the owners reported intermittent loud breathing and chronic coughing.

General examinations (temperature, heart and respiratory rates etc.), respiratory examinations (oropharyngeal examination, tracheal palpation, tracheal and thoracic auscultation, and characterization of breathing sounds and type) and routine hematological testing of the dogs were carried out. Lateral thoracic and cervical radiographs of the dogs were performed to evaluate the respiratory system.

All dogs were sedated with xylazine $\mathrm{HCl}(1.5 \mathrm{mg} / \mathrm{kg}$, im.) and induced with a combination of ketamine $\mathrm{HCl}(5 \mathrm{mg} / \mathrm{kg})$ and diazepam $(0.5 \mathrm{mg} / \mathrm{kg})$. Orotracheal intubations of the dogs were performed, and an assistant held the dogs' heads in extension to facilitate the insertion of an endoscope. The vital parameters of the dogs were routinely checked during anesthesia and endoscopic examinations.

A fiberoptic flexible endoscope (Karl Storz ${ }^{\circledR}$, Germany) with a diameter of $5.2 \mathrm{~mm}$ and a length of $85 \mathrm{~cm}$ was used for tracheobronchoscopic examinations. The endoscope was inserted through the trachea via the endotracheal tube, and tracheobronchoscopic examinations were performed and recorded with a digital recorder system (Tele Pack Vet X, Karl Storz ${ }^{\circledR}$, Germany). Tracheobronchial examinations of the dogs focused on the mucosal surfaces of the entire intratracheal lumen (from laryngeal ring to carina, and right and left main bronchus). Endoscopic findings were evaluated according to the following criteria, as reported previously (13): mucosal surfaces $(0$ - smooth, 1 - mildly irregular,
2 - markedly irregular), mucosal color $(0$ - pale, 1 - erythematous; 2 - both white and red areas), prominence of vessel $(0$ - no visible vessels, 1 - visible vessels, 2 - large distended blue vessels, 3 - very large protruding tortuous vessels), chondral ring abnormalities of the trachea $(0-$ no abnormalities, 1 - mild alteration of ring shapes, 2 - moderate and irregular chondral rings, 3 - severe chondral ring abnormalities), presence of bronchial collapse $(0-$ no decrease, 1 - mild collapse, 2 - moderate collapse, 3 - severe collapse) and presence of tracheal collapse (1 - grade I, 2 - grade II, 3 - grade III, 4 - grade IV). These abnormalities were reviewed.

Following the tracheobronchial examinations, a BAL procedure was performed for sampling as reported previously $(13,14)$. The bronchial tree was flushed with $0.5 \mathrm{ml}$ of sterile saline $(0.9 \%)$ (per $\mathrm{kg}$ bodyweight). The sample was aspirated and put into a sterile tube for cytological examination, bacteriological culture, and antibiotic susceptibility testing.

Slides were prepared using precipitates from the BAL samples, which were stained by Diff-quick stain, and selected areas were then evaluated for cellular components (epithelial cells, neutrophils, macrophages, etc.) and bacteria.

BAL fluid samples were cultured for bacteria and yeasts with 3 pair on $5 \%$ defibrinated sheep blood (one incubated aerobically, one micro-aerobically and the third anaerobically), MacConkey (MCA, Oxoid) agar and Sabouraud dextrose (SAD, Oxoid) agar (incubated aerobically at $37^{\circ} \mathrm{C}$ and $28^{\circ} \mathrm{C}$ ), respectively. Pleuropneumonia-like organism (PPLO) base agar (Oxoid) was used for Mycoplasma isolation with incubation at $35^{\circ} \mathrm{C}$ in $5 \% \mathrm{CO}_{2}$. After incubation for 48-72 hours, plates were examined for bacterial growth. Standard biochemical methods and commercial miniaturized identification systems (BBL Crystal Panel ${ }^{\mathrm{TM}}$ ) were used for identification of pure cultures. Furthermore, the plates were incubated for an extended period for observation of slower-growing Mycoplasma and mycotic microorganisms.

In accordance with the standards of the Clinical Laboratory Standards Institute (CLSI) (3), bacterial susceptibility testing was performed using the Kirby-Bauer disc diffusion procedure. For E. coli isolates, 12 antibiotics were used as reported previously (12): sulphamethoxazole/trimethoprim 19: 1 (SXT, $25 \mu \mathrm{g})$, ceftriaxone (CRO, $30 \mu \mathrm{g})$, gentamicin $(\mathrm{CN}, 10 \mu \mathrm{g})$, ampicillin (AMP, $10 \mu \mathrm{g})$, ceftazidime (CAZ, $30 \mu \mathrm{g}$ ), tobramycin (TOB, $10 \mu \mathrm{g}$ ), amoxicillin clavulanic acid (AMC, $30 \mu \mathrm{g})$, amikacin (AK, $30 \mu \mathrm{g})$, ciprofloxacin (CIP, $5 \mu \mathrm{g}$ ), cloxacillin (OB, $5 \mu \mathrm{g})$, cefoperazone (CFP, $75 \mu \mathrm{g}$ ), and cefotaxime (CTX, $30 \mu \mathrm{g}$ ). The reference bacterial strains of E. coli (ATCC 25922) and Staphylococcus aureus (ATCC 25923) were used as quality control strains following the recommendations of CLSI.

Ampicillin-sulbactam (20 mg/kg, im, bid for 5 days), which has been recommended for treatment of respiratory system diseases (13), was administered to all dogs prior to the completion of antibiotic susceptibility testing. Antibiotic therapy was continued according to susceptibility results in all dogs.

Total scores from tracheobronchoscopic findings, representing endoscopic diagnoses, were compared statistically in terms of significance $(\mathrm{p}<0.05)$ using one-way ANOVA (SPSS 23.0, IBM ${ }^{\circledR}$, USA). 
Tab. 1. Signalments of the dogs, tracheobronchoscopic findings, endoscopic diagnosis, cytological and microbiological results of BAL samples

\begin{tabular}{|c|c|c|c|c|c|c|c|c|c|c|c|}
\hline \multirow{2}{*}{$\begin{array}{c}\text { Case } \\
\text { no }\end{array}$} & \multirow{2}{*}{ Signalments } & \multicolumn{7}{|c|}{ Tracheobronchoscopic findings } & \multirow{2}{*}{$\begin{array}{l}\text { Endoscopic } \\
\text { diagnosis }\end{array}$} & \multirow{2}{*}{$\begin{array}{l}\text { Cytological } \\
\text { results }\end{array}$} & \multirow{2}{*}{$\begin{array}{l}\text { Microbiological } \\
\text { results }\end{array}$} \\
\hline & & MS & MC & PVA & CRA & $\mathrm{BC}$ & $\mathrm{TC}$ & Total no & & & \\
\hline 1 & $\begin{array}{l}\text { German shepherd, } \\
8 \text { years old, } 42 \text { kg }\end{array}$ & 2 & 1 & 2 & 2 & 0 & 1 & 8 & tracheal collapse & $\begin{array}{l}\text { minimal epithelial } \\
\text { cells }\end{array}$ & - \\
\hline 2 & $\begin{array}{l}\text { German shepherd, } \\
4 \text { years old, } 36 \mathrm{~kg}\end{array}$ & 1 & 2 & 1 & 1 & 1 & 0 & 6 & $\begin{array}{l}\text { right bronchial } \\
\text { collapse }\end{array}$ & $\begin{array}{l}\text { minimal epithelial } \\
\text { cells }\end{array}$ & E. coli \\
\hline 3 & $\begin{array}{l}\text { Terrier, } \\
7 \text { years old, } 12 \text { kg }\end{array}$ & 1 & 1 & 1 & 2 & 0 & 1 & 6 & tracheal collapse & $\begin{array}{l}\text { minimal epithelial } \\
\text { cells }\end{array}$ & E. coli \\
\hline 4 & $\begin{array}{l}\text { Golden retriever, }, \\
13 \text { years old, } 28 \text { kg }\end{array}$ & 1 & 0 & 1 & 2 & 1 & 0 & 5 & $\begin{array}{l}\text { right bronchial } \\
\text { collapse }\end{array}$ & $\begin{array}{l}\text { minimal epithelial } \\
\text { cells and neutrophils }\end{array}$ & - \\
\hline 5 & $\begin{array}{l}\text { Chow chow, } \\
8 \text { months old, } 22 \text { kg }\end{array}$ & 0 & 2 & 1 & 1 & 0 & 1 & 5 & tracheal collapse & $\begin{array}{l}\text { minimal epithelial } \\
\text { cells and neutrophils }\end{array}$ & - \\
\hline 6 & $\begin{array}{l}\text { Mixed breed, } \delta \text {, } \\
7 \text { years old, } 19 \text { kg }\end{array}$ & 1 & 2 & 3 & 1 & 0 & 1 & 8 & tracheal collapse & $\begin{array}{l}\text { minimal epithelial } \\
\text { cells }\end{array}$ & - \\
\hline 7 & $\begin{array}{l}\text { Presa canario, } \hat{\partial} \text {, } \\
7 \text { months old, } 35 \text { kg }\end{array}$ & 1 & 1 & 1 & 1 & 0 & 1 & 5 & tracheal collapse & $\begin{array}{l}\text { keratinized epithelial } \\
\text { cells }\end{array}$ & - \\
\hline 8 & $\begin{array}{l}\text { Mixed breed, } \delta \text {, } \\
6 \text { years old, } 25 \mathrm{~kg}\end{array}$ & 1 & 2 & 3 & 1 & 1 & 1 & 9 & $\begin{array}{l}\text { tracheal and right } \\
\text { bronchial collapse }\end{array}$ & $\begin{array}{l}\text { minimal epithelial } \\
\text { cells }\end{array}$ & - \\
\hline
\end{tabular}

Explanations: MS - mucosal surfaces ( 0 - smooth, 1 - mildly irregular, 2 - markedly irregular $)$; MC - mucosal color $(0$ - pale, 1 - erythematous, 2 - both white and red areas); PVA - prominence of vessels ( 0 - no visible vessels, 1 - visible vessels, 2 - large distended blue vessels, 3 - very large, protruding tortuous vessels); CRA - chondral ring abnormalities of the trachea ( 0 - no abnormalities, 1 - mild alteration of ring shapes, 2 - moderate and irregular chondral rings, 3 - severe chondral ring abnormalities); BC - bronchial collapse ( 0 - no decrease, 1 - mild collapse, 2 - moderate collapse, 3 - severe collapse); TC - tracheal collapse ( 1 - grade I, 2 - grade II, 3 - grade III, 4 - grade IV)

\section{Results and discussion}

The signalments of the dogs are given in Tab. 1. General examinations revealed no remarkable findings in the vital parameters. Hematological values of the dogs were also within normal reference ranges. Tracheal palpations of the dogs were reflective of tracheal disease, but there were no notable clinical signs related to respiratory system disease in these cases. Breathing sounds were not recorded during examina-

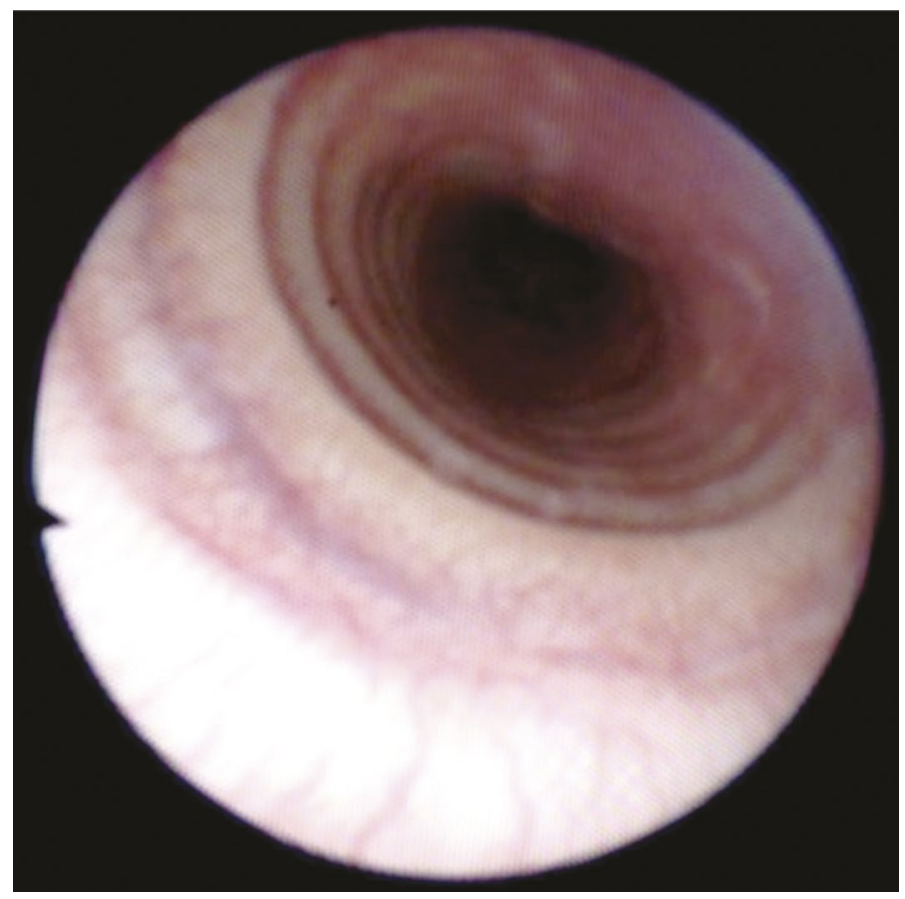

Fig. 1. Tracheoscopic view of the grade 1 tracheal collapse in case 6 tions, but all dogs were observed to have intermittent coughing. On radiographs, the tracheal ring contours were more distinctive in cases 3 and 6 , with no other radiologic abnormalities noted in the thoracic or cervical radiographs of all cases.

Tracheobronchoscopic findings and endoscopically diagnosed pathologies are reported in Tab. 1. On the basis on these results, tracheal collapse $(n=5)$ (Fig. 1), right bronchial collapse $(n=2)$ (Fig. 2), and concur-

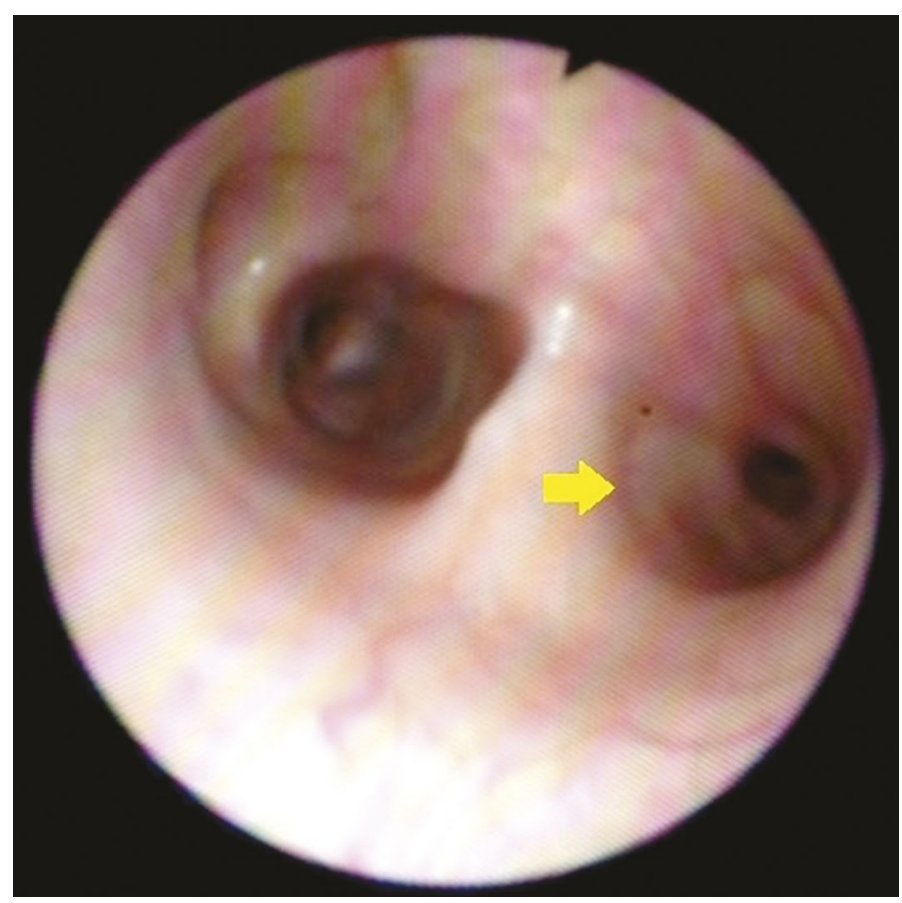

Fig. 2. Bronchoscopic view of the right bronchial collapse (arrow) in case 4 
rent tracheal and right bronchial collapse $(n=1)$ were diagnosed in these cases.

Cytologically, the cellularity of the preparations was very low. The preparations from cases 1, 2, 3, 6 and 8 contained minimal epithelial cells. There were also minimal numbers of epithelial cells and neutrophilic leukocytes in the preparations from cases 4 and 5 . Keratinized epithelial cells (pharyngeal epithelia) were noted in case 7 , but no bacterial microorganisms were detected in the cytological preparations.

Microbiologically, E. coli was cultured from cases 2 and 3, and these isolates were susceptible to only sulfamethoxazole/trimethoprim at $19: 1$. Given these antibiotic susceptibility results, sulfamethoxazole/ trimethoprim was administered to cases 2 and 3 as a treatment protocol. However, this antibiotic was also administered to the other cases prophylactically.

Statistically, there was no significant difference between the endoscopic diagnoses, according to total scores of tracheobronchoscopic findings ( $p=0.213$ ).

The present study showed that tracheobronchoscopic examination combined with cytological and microbiological evaluations in cases of tracheal and/or bronchial collapse provided advantages for clinicians during the diagnostic work-up in dogs suffering from coughing. Clinical features of airway collapse are not predictive due to the severity of the collapse $(8,15)$. It has been noted that respiratory distress, exercise intolerance, and chronic coughing are documented in dogs with airway collapse $(1,2,4)$. Although there is no gold standard for clinical diagnosis of lower respiratory system disease $(8,12)$, endoscopic examinations of the respiratory tract have the advantage of clarifying the exact diagnosis in these cases $(2,8,15)$. Historical, physical, hematological, radiographic and tracheobronchoscopic findings known to be compatible with respiratory system disease are often nonspecific $(1,4,8,10,12)$. In our study, all cases had reflective tracheal palpation and intermittent chronic coughing, but their vital and hematological parameters showed no remarkable abnormalities. Thoracic radiography is not sensitive in detecting lesions $(1,8,10)$. Abnormalities detected radiographically are rarely specific for a single disease process $(6,15)$. Thus, if tracheal collapse is suspected in a case, fluoroscopic examination should be made to grade the disease $(1,8,10)$. Furthermore, radiological scoring is considered to be highly subjective, and diagnosis of respiratory system pathologies is not possible with radiological assessment alone (8). In the present study, radiological findings were generally nonspecific. However, tracheal ring contours were more distinctive in radiographs.

Diagnostic techniques used in small animals to evaluate the respiratory system include thoracic radiography, tracheal wash, tracheobronchoscopy, bronchial brushing or biopsy, transthoracic lung aspiration and lung biopsy $(1,4,6,12,14)$. Tracheobronchoscopy is an important procedure for evaluating respiratory diseases in dogs with abnormal breathing patterns and chronic coughing $(1,2,4,9,10,14,15)$, and airway collapse can be graded and documented only by using endoscopic examinations $(8,14)$. Respiratory tract visualization with an endoscope makes it possible to assess the upper and lower respiratory mucosa $(8,14)$, evaluate dynamic airway changes $(2,11$, 14) and collect deep respiratory samples for cytology, histology and culture $(5-7,9,11-14)$. In parallel to these observations $(5,11,12,14)$, in the current study, detailed tracheobronchoscopic examinations of dogs with tracheal and/or bronchial collapse and cytological and microbiological results of BAL fluids were evaluated. Scoring systems based on predefined macroscopic findings are used to improve objectivity in the assessment of the type and severity of bronchial lesions $(2,8,11,15)$. As reported previously $(13)$, in this study, a scoring system was used to compare the tracheobronchoscopic data of the cases. According to these results, diagnoses of the cases were made, and the severity of the disease was evaluated. Irregularity of the tracheal and bronchial mucosa may be related to the presence of subclinical chronic inflammatory changes (11). Chondral ring abnormalities are more common in dogs with tracheal collapse $(2,8)$. The presence of large, protruding, and pulsatile vessels is a distinctive finding in older dogs and is considered to be secondary to bronchial wall alterations and vascular remodeling (11). A markedly irregular mucosal surface was encountered in one case with tracheal collapse. Prominent appearance of vessels in the tracheal lumen was severe in cases 6 and 8, and all cases had mild or moderate chondral ring abnormalities. The tracheobronchoscopic examination findings were used to calculate total scores, and statistical comparison of these scores revealed that there were no significant differences between the cases.

BAL is the most recognized diagnostic technique for sampling from the respiratory tract. In this technique, sterile saline is instilled into an airway in sufficient volume to bathe the airways $(1,6,8,12-14)$. The BAL fluid may be analyzed cytologically, microbiologically, biochemically and immunologically $(5,6,9,12,14)$. Although transtracheal aspiration and transtracheal wash specimens are also widely used $(12,14)$, infectious organisms, including bacteria and fungi, are more often found in the cytology of BAL samples (9). Here, BAL was performed for cytological and microbiological examination of the cases with tracheal and/or bronchial collapse. Identification of normal reference ranges is impeded by a lack of standardization (6). Epithelial and inflammatory cells in BAL fluid are considered to be the most sensitive indicators of an inflammatory response $(1,5)$. Total nucleated cell counts should be less than 500 nucleated cells/ $\mu 1$ for healthy dogs. In chronic inflammation, relatively non-specific 
findings, such as chronic active inflammation, may occur. Macrophages should be examined specifically for the presence of bacteria and other microorganisms (6). Bacteria and intracellular bacteria are not always identified in cytological preparations of BAL fluids $(9,12)$. As noted above, our cytological results were non-specific with a lack of standardization. Cytological examination and bacterial cultures of BAL fluids may aid in the diagnosis of diseases of the lower respiratory tract, but this technique is not very sensitive for diagnosis of respiratory system diseases $(8,12)$. In addition, mucosal biopsies obtained by endoscopy may show no signs of inflammation $(11,12)$. Cytological results in these cases were not compatible with inflammation or infection. Our cytological examination did not reveal any substantial cellularity indicative of possible inflammation (12). Epithelial cells, keratinized epithelial cells (from oro-pharyngeal mucosa) and minimal neutrophilic leukocytes were detected in the cytological preparations.

The tracheobronchial tree of healthy dogs has not always been shown to be sterile. Normal tracheal flora is thought to be the result of continuous minor aspiration, contamination from the upper respiratory tract, or a combination of both (12). Bacteria are commonly isolated from dogs with tracheal collapse (7). Various bacterial agents such as E. coli, Staphylococcus epidermidis, Klebsiella pneumoniae, Mycoplasma spp. Pseudomonas spp. and Enterobacter cloaca are typically cultured from dogs with respiratory system disorders $(5,12)$. In this study, cytological preparations did not reveal any bacteria, but $E$. coli was isolated in cultures from case 2 (bronchial collapse) and case 3 (tracheal collapse). Treatment undertaken on the basis of bacterial sensitivity testing is more specific than empirical treatment in dogs. Culture and antibiotic susceptibility testing of bronchopulmonary secretions should be performed prior to administration of antibiotics (12). Until the culture and antibiotic susceptibility analysis were completed, an empirical antibiotic regimen had been applied to all dogs. However, following the culture and antibiotic susceptibility results, sulfamethoxazole/trimethoprim was administered to cases 2 and 3.

In conclusion, our study results suggest that bronchial collapse, rather than tracheal collapse, should only be diagnosed by tracheobronchoscopic examinations. Cytological and microbiological analysis of BAL fluids needs to be performed to evaluate the health status of the respiratory tract, but these analyses do not always provide valuable data for clinical practitioners.

\section{References}

1. Adamama-Moraitou K. K., Pardali D., Day M. J., Prassinos N. N., KritsepiKonstantinou M., Patsikas M. N., Rallis T. S.: Canine bronchomalacia: a clinicopathological study of 18 cases diagnosed by endoscopy. Vet. J. 2012, 191, 261-266.

2.Bottero E., Bellino C., De Lorenzi D., Ruggiero P., Tarducci A., D'Angelo A., Gianella P.: Clinical evaluation and endoscopic classification of bronchomalacia in dogs. J. Vet. Intern. Med. 2013, 27, 840-846.
3. Clsi: Performance standards for antimicrobial susceptibility testing; twentieth informational supplement, CLSI document M100-S20, Clinical and Laboratory Standards Institute, Wayne, PA, USA 2010.

4. Fossum T. W.: Small animal surgery. Mosby, USA 2002

5. Gonul R., Koenhemsi L., Or M. E., Uysal A., Sonmez K., Gurel A., Bagcigil A. F., Ozgur N. Y., Yardibi H., Altunatmaz K.: Biochemical and cytological analysis of bronchoalveolar lavage (BAL) fluid and effects on arterial blood gases in dogs with lower respiratory airway disease. Revue Med. Vet. 2010, $161,233-238$.

6. Hawkins E. C., DeNicola D. B., Kuehn N. F.: Bronchoalveolar lavage in the evaluation of pulmonary disease in the dog and cat. J. Vet. Intern. Med. 1990, 4, 267-274.

7. Johnson L. R., Fales W. H.: Clinical and microbiologic findings in dogs with bronchoscopically diagnosed tracheal collapse: 37 cases (1990-1995). JAVMA 2001, 209, 1247-1250

8. Johnson L. R., Pollard R. E.: Tracheal collapse and bronchomalacia in dogs: 58 cases (7/2001-1/2008). J. Vet. Intern. Med. 2010, 24, 298-305.

9. Johnson L. R., Queen E. V., Vernau W., Sykes J. E., Byrne B. A.: Microbiologic and cytologic assessment of bronchoalveolar lavage fluid from dogs with lower respiratory tract infection: 105 cases (2001-2011). J. Vet. Intern. Med. 2013, 27, 259-267.

10. Johnson L. R., Singh M. K., Pollard R. E.: Agreement among radiographs, fluoroscopy and bronchoscopy in documentation of airway collapse in dogs. J. Vet. Intern. Med. 2015, 29, 1619-1626.

11. Mercier E., Bolognin M., Hoffman A. C., Tual C., Day M. J., Clercx C.: Influence of age on broncoscopic findings in healthy beagle dogs. Vet. J. 2011, 187, 225-228.

12. Peeters D. E., McKiernan B. C., Weisiger R. M., Schaeffer D. J., Clercx C.: Quantitative bacterial cultures and cytological examination of bronchoalveolar lavage specimens in dogs. J. Vet. Intern. Med. 2000, 14, 534-541.

13. Salci H., Kahya S., Çetin M., AkkoçA., Bayram A. S.: Evaluation of pulmonary infection risk in dogs with pulmonary contusion. Kafkas Üniv. Vet. Fak. Derg. 2017, 23, 613-620.

14. Tams T. R.: Small animal endoscopy. Mosby, USA 1999, p. 378-387.

15. Tappin S. W.: Canine tracheal collapse. J. Small Anim. Pract. 2015, 57, 9-17.

16. Woods K. S., Defarges A. M. N., Abrams-Ogg A. C. G., Viel L., Brisson B. A., Bienzle D.: Comparison of manual and suction pump aspiration techniques for performing bronchoalveolar lavage in 18 dogs with respiratory tract disease. J. Vet. Intern. Med. 2014, 28, 1398-1404.

Corresponding author: Hakan SALCI, DVM, PhD, Assoc. Prof. Dr., Uludag University, Faculty of Veterinary Medicine, Department of Surgery, Gorukle Campus, 16059, Bursa, Turkey; e-mail: hsalci@uludag.edu.tr 